\title{
Use of disability adjusted life years in health planning: a plea for caution
}

\section{There are more cost-effective alternative approaches to use of DALYs}

\author{
Saroj Jayasinghe ${ }^{1}$, Nalaka Mendis ${ }^{2}$ and Reidar Lie $^{3}$
}

In the recent past there has been an increasing interest among economists and policy makers on the links between health and development. For example, the World Bank dedicated its 1993 World Development Report to the topic of investing in health (1). These efforts require quantifying the societal, individual and economic burdens of illness and disease (2). An example of a recently promoted methodology in quantifying burden is disability adjusted life years (DALYs), which has promoted a fresh look at methods of measuring burden of disease (BOD). This is conceptually similar to previous attempts at quantifying quality of life (with different disorders and diseases) during an individual's life span, eg. quality adjusted life years (QALYs) $(3,4)$. Such indices enable the comparison of conditions with different degrees of disability and premature death, which is not possible with morbidity and mortality data. They could also be used to quantify interventions, as savings in DALYs or QALYs per cost unit, which is a method of assessing cost effectiveness between interventions for different disorders. QALYs had its advocates and there were several attempts to use it in resource allocation (5).

More recently, the attention of policy makers has largely focused on DALYs, partly the result of it being supported by donor organisations such as the WHO and the World Bank. Several countries and organisations are using DALYs to identify health priorities and costeffective interventions and to allocate resources for health (1). A recent advance has been the use of Disability Adjusted Life Expectancy by the World Health Report 2000, which looks at the gains in health status for different interventions (3).

In Sri Lanka, too, researchers and policy makers are urging the use of BOD and DALYs as it provides objective information to health planners to monitor and evaluate intervention programs (4). Surprisingly, no such attempts were made previously with QALYs, which has been in existence for several decades.

This paper summarises four areas of concern in the management and application of DALY in health planning, which are relevant to policy makers in Sri Lanka. They include conceptual issues, ethical issues, process used in developing DALYs and their use in the practical situation.

\section{Conceptual issues}

There are 3 main areas of concern:

a) DALY attempts to aggregate disabilities suffered by individuals as a result of a disease, disorder or injury. Hence the focus is more on diseases rather than their socio-economic determinants and socio-cultural factors which are relevant to policy makers (and probably less so for clinicians). For example, poverty, income inequality, social networks and other factors such as social capital, are known to influence disease prevalence, severity and outcome (8). Using DALYs as a tool to prioritise interventions would tend to reinforce the medicalised view of health, based on diseases and disorders. The role of social, environmental and behavioural factors that contribute to diseases, illnesses and disabilities would attract less attention and emphasis in health policies. As a result, interventions tend towards disease specific areas rather than to environmental and social issues.

b) DALYs measure functional limitations (activities related to daily living) and premature mortality (6). Handicap (or disadvantage) and the social context are not included in the description of disability classes and weighting (9). QALYs includes social activity in its computation (5). For example, in a condition such as obstetric fistula the stigma, suffering and social consequences would not be captured with DALYs (9). A more common situation in Sri Lanka are the quadriplegics or wheel-chair bound disabled persons. They are less likely to gain employment as a result of discrimination, to be mobile for lack of wheel-chairs and gain physical access to buildings for absence of ramps. DALYs capture poorly such limitations in social functioning.

c) DALYs and other measures of quality of life can be criticised for placing a strong emphasis on functional and role limitations, according to which the life of a paraplegic would be rated low although some authorities take the philosophical position that only the individual can rate quality of life (4).

\section{Ethical issues}

In developing DALYs, different weights are given to different age groups. This raises fundamental ethical issues (10). Even though a new method is being adopted in the later versions (patient Trade-Off Technique), this is also being criticised, as it confuses the value of health with the value of life (10). Should we value life differently at different age groups? Do Sri Lankans, with their extended

\footnotetext{
${ }^{1}$ Departments of Clinical Medicine and ${ }^{2}$ Psychological Medicine, Faculty of Medicine, University of Colombo; ${ }^{3}$ Professor of Philosophy, University of Bergen, Norway. (Correspondence: SJ, tel: 01 695300, 01 697094. Submitted 14 September 2001, accepted 2 February 2002).
} 
family structure, value their elderly less than the young? Are disabled persons less valuable to us? The authors of DALYs have done sensitivity analysis on different age weights (and discount rates) and shown that their formula used to estimate DALYs is robust (11). Even if one assumes that these are applicable across cultures, that does not negate the ethical issues of giving different values to life span and between individuals.

\section{Process used to develop DALYs}

The principle criticisim against DALYs is that only the views of experts, and not lay persons, are included in the weighting.

Attempts at comparing disability ranking by respondents from different countries for the same condition have shown wide disparities in results (12). Thus the relevance of weights and ranking of disability to the vast majority of people in the poorer developing countries and their cultural beliefs, social structures and relationships need to be taken into account. One option is to develop our own culturally sensitive weighting system of the common disorders, diseases and their determinants.

\section{Use of DALYs in practice}

The practical value of DALYs in planning for Sri Lanka is further complicated by defects in the system of reporting morbidity and mortality. For example, determinants and contributing factors to death and disability, such as alcohol and tobacco, are rarely noted as contributing to death in death certificates. Their impact on mortality and morbidity figures (eg. alcohol in road traffic accidents or tobacco in ischaemic heart disease), are not quantified, resulting in the underestimation of burden as a result of alcohol and tobacco use. Our medical records system is in a very shabby state and does not capture out-patient data, data from the private sector and community morbidity. Before attempting to estimate the BOD using DALYs with existing data, it is essential to ensure that an acceptable medical records system is in place.

Even with reliable data there are practical problems of using DALYs in resource allocation. Let us consider two diseases that have different burdens (eg. epilepsy with 202 DALY/100 000 and asthma with 901.9 DALY/100 000 (7). To simplify, let us assume that they have equal prevalence and that there are equally cost-effective interventions for each condition (eg. specialised epilepsy clinic versus specialist asthma clinic). In other words, using the same quantum of resources, either of these clinics would reduce the burden by the same magnitude (ie. same gain of DALYs per rupees spent). In this example the larger burden is caused by asthma and hence the priority intervention should logically be to establish an asthma clinic. What does this mean in practical terms? Would the Department of Health open an asthma clinic instead of allocating funds for an epilepsy clinic? It becomes still more difficult if both an asthma clinic and an epilepsy clinic already exist. In this instance, how could one use BOD and DALYs to apportion scarce resources? How useful is the information on BOD to establish a general medical clinic that manages both epilepsy and asthma?

\section{Conclusions}

We have discussed certain limitations concerning the computation and application of DALYs in health planning. These need to be carefully considered by policy makers who evaluate them for use in Sri Lanka. Already the need to use of DALYs and BOD in health policy is being emphasised in various forums in Sri Lanka $(7,13)$. Experience has shown that such measurements of quality of life have failed to make a visible impact on resource allocation and policies in Sri Lanka. But DALYs may survive the test of time, especially because the World Bank, and now the WHO, back it though the chief architects of this trend in both institutions appear to be the same group of researchers.

We also should be aware of our own achievements in health, accomplished by using alternative approaches of social welfare and preventive health to achieve good health. For example, the World Bank in its World Development Report, using the DALYs methodology suggests that a "minimum package" of public health and clinical intervention would reduce disease burden in low-income developing countries by about a quarter. They estimated the annual cost for such an intervention to be US\$ 12 for every person (1). Sri Lanka surpassed the levels of health status attainable by the DALYs based World Bank prescription, with an annual investment of a mere US\$ 8 per person. This indicates that the efficiency of our formula was more than DALYs based allocations would offer against specific diseases. It has more to do with the tackling of social issues such as universal adult franchise, free education, a good transport network and health care delivery to village levels, especially primary health care. To this we should add an extensive and relatively comprehensive state health system delivered at zero charge to the user, a point that seems to irk many proponents of a health care market. The world, and the World Bank, would probably end up wiser if they begin to learn from us, rather than enforcing yet another tool for misallocating resources.

\section{Acknowledgements}

We acknowledge the financial assistance from the Health Development Research Program of the University of Colombo.

\section{References}

1. TheWorld Development Report 1993. Investing in Health, Washington: The World Bank, 1993.

2. Murray CJL, Lopez AD. Global comparative assessments in the health sector. Geneva: WHO, 1994.

3. Guyatt GH, Cook DJ. Health status, Quality of life and the individual. JAMA 1994; 272: 630-1.

4. Gill TM, Feinstein. A critical appraisal of the quality of Qualityof-Life measurements. JAMA 1994; 272: 619-26. 
5. Loomes G, Mckenzie L. The use of QALYs in health care decision making. Social Science and Medicine 1989; 289: 299-308

6. World Health Organization, The World Health Report 2000, Health systems: improving performance. Geneva: WHO, 2000.

7. Wijewardena K, Spohr M. An attempt to measure burden of disease using disability adjusted life years in Sri Lanka. Ceylon Medical Journal 2000; 45: 110-15.

8. Lynch JW, Kaplan GA, Salonen JT. Why do poor people behave poorly? Variation in adult health behaviours and psychosocial characteristics by stages of the socioeconomic lifecourse. Social Science and Medicine 1997; 44: 809-19.

9. AbouZahr C, Vaughan JP. Assessing the burden of sexual and reproductive ill-health: questions regarding the use of disability- adjusted life years. Bulletin of the World Health Organization 2000; 78: 655-65.

10. Arnesan T, Nord E. The value of DALY life: problem with ethics and validity of disability adjusted life years. British Medical Journal 1999; 319: 1423-5.

11. Murray CJL, Lopez AD. The utility of DALYs for public health policy and research: a reply. Bulletin of the World Health Organization 1997; 75: 377-8.

12. James KC, Foster SD. Weighing up disability. Lancet 1999; 354: 87-8.

13. Summary Report on "Preliminary Workshop on the Technical Assistance Programme under the Policy and Human Resource Development (PHRD) grant of the World Bank". Ministry of Health. Sri Lanka, 2001. 